\title{
Universal Point Subsets for Planar Graphs
}

\author{
Patrizio Angelini ${ }^{1}$, Carla Binucci ${ }^{2}$, William Evans ${ }^{3}$, Ferran Hurtado ${ }^{4}$, \\ Giuseppe Liotta ${ }^{2}$, Tamara Mchedlidze ${ }^{5}$, Henk Meijer $^{6}$, and Yoshio Okamoto ${ }^{7}$ \\ 1 Roma Tre University, Italy \\ ${ }^{2}$ University of Perugia, Italy \\ ${ }^{3}$ University of British Columbia, Canada \\ ${ }^{4}$ Universitat Politécnica de Catalunya, Spain \\ ${ }^{5}$ Karlsruhe Institute of Technology, Germany \\ ${ }^{6}$ Roosevelt Academy, Netherlands \\ 7 University of Electro-Communications, Japan
}

\begin{abstract}
A set $S$ of $k$ points in the plane is a universal point subset for a class $\mathcal{G}$ of planar graphs if every graph belonging to $\mathcal{G}$ admits a planar straight-line drawing such that $k$ of its vertices are represented by the points of $S$. In this paper we study the following main problem: For a given class of graphs, what is the maximum $k$ such that there exists a universal point subset of size $k$ ? We provide a $\lceil\sqrt{n}\rceil$ lower bound on $k$ for the class of planar graphs with $n$ vertices. In addition, we consider the value $F(n, \mathcal{G})$ such that every set of $F(n, \mathcal{G})$ points in general position is a universal subset for all graphs with $n$ vertices belonging to the family $\mathcal{G}$, and we establish upper and lower bounds for $F(n, \mathcal{G})$ for different families of planar graphs, including 4-connected planar graphs and nested-triangles graphs.
\end{abstract}

\section{Introduction}

A classic result in graph theory states that every planar graph $G=(V, E)$ can be drawn without crossings on the plane using some set $S$ of points as vertices, and straight-line segments with endpoints in $S$ to represent the edges [7, 16,21]. However, not every set $S$ with $n=|S|=|V|$ is suitable for such a representation; for example, the drawing is impossible if $G$ is a maximal planar graph with $n>3$ vertices and $S$ is in convex position, because in this case $|E|=3 n-6$ while at most $2 n-3$ segments can be drawn between points of $S$ without crossings. In fact, Cabello [4] proved that deciding whether there is a planar straight-line drawing of $G=(V, E)$ using a point set $S$ with $|S|=|V|$ is an NP-complete problem.

As the number of combinatorially different sets of $n$ points is finite [8], it is obvious that there exist some adequate yet huge sets of points $U$, such that given any planar graph $G$ with $n$ vertices, some $n$-subset of $U$ admits a planar straight-line drawing of $G$. The challenge though, is to find sets $U$ with that property, yet as small as possible. We define next this problem more precisely.

A set $U$ of $k$ points in the plane is a universal point set if every planar graph with $n$ vertices admits a planar straight-line drawing whose vertices are a subset of the points of $U$. From the literature it is known that if $U$ is a universal point set for planar graphs 
then $1.235 n \leq|U| \leq 8 n^{2} / 9$. Indeed, Kurowski [10] proved that the size of $U$ requires at least 1.235n points, while de Fraysseix, Pach, and Pollack [5], Schnyder [15] and Brandenburg [3] showed that a $O(n) \times O(n)$ grid of points is a universal point set.

This topic has been a very active area of research since it was introduced, and several variations have been considered. For example, one can restrict the family of graphs to be represented. In this sense, Gritzman, Mohar, Pach and Pollack [9] proved that every set of $n$ distinct points in the plane in general position (no three collinear) is universal for the class of outerplanar graphs with $n$ vertices.

In this paper we introduce and study the notion of a universal point subset. A set $S$ of $k$ points is a universal point subset for a class $\mathcal{G}$ of planar graphs if every graph in $\mathcal{G}$ admits a planar straight-line drawing such that $k$ of its vertices are represented by the points of $S$.

In Section 2 we prove that a particular, very flat convex chain of $\lceil\sqrt{n}\rceil$ points is a universal point subset for the class of (maximal) planar graphs with $n$ vertices.

For a certain subfamily of 4-connected planar graphs we have been able to obtain a bound that is stronger in a particular sense, namely that every set of $\left\lceil\frac{\lg n}{4}\right\rceil$ points in general position is a universal point subset for all the graphs with $n$ vertices in this family. Inspired by this result, we consider in Section 3 the value $F(n, \mathcal{G})$ such that every set of $F(n, \mathcal{G})$ points in general position is a universal subset for the planar graphs with $n$ vertices belonging to $\mathcal{G}$. It is trivial to prove that every set of 1,2 , or 3 points in general position is a universal point subset for every planar graph and every value of $n$ (Tutte's algorithm $[19,20])$. On the other hand there exists a set of 4 points in general position that is not a universal point subset for planar graphs having $n=5$ vertices.

We show lower and upper bounds for $F(n, \mathcal{G})$ for different families of planar graphs. In particular, we show that every set of 4 points in general position is a universal point subset for all planar graphs with at least 6 vertices and, on the other hand, we show that there exists a set of $2\left\lceil\frac{n}{3}\right\rceil+2$ points in convex position that is not a universal subset for the class of planar graphs. In other words, we prove that $4 \leq F(n, \mathcal{G}) \leq 2\left\lceil\frac{n}{3}\right\rceil+1$, for all $n \geq 6$, when $\mathcal{G}$ is the class of all planar graphs. In addition, we improve the lower bound and the upper bound for some subfamilies of planar graphs; specifically, we study the case that $\mathcal{G}$ is the class of 4-connected planar graphs whose outer face is a quadrilateral, and the case that $\mathcal{G}$ is the class of nested-triangles graphs.

We conclude in Section 4 with some remarks and open problems. In that section we also briefly discuss the relationships between our problem and the related allocation problem that has been the subject of recent studies (see, e.g. [11, 13]).

\section{Definitions and Notation}

Point sets. A set $S$ of points is in general position if no three points are collinear. The convex hull $C H(S)$ of $S$ is the point set obtained as a convex combination of the points of $S$. If no point is in the convex hull of the others, then $S$ is in convex position. A set $S$ in convex position is one-sided if it can be rotated in such a way that the leftmost and rightmost points are consecutive in the convex hull.

Graphs. We denote by $(u, v)$ both an undirected and a directed edge, in the latter case meaning the edge is directed from $u$ to $v$. Also, we use the term triangle to denote 
both a 3-cycle and its drawing. A graph $G=(V, E)$ is planar if it has a drawing $\Gamma$ without edge crossings. Drawing $\Gamma$ splits the plane into connected regions called faces; the unbounded region is the outer face and the other faces are the internal faces. The cyclic ordering of edges around each vertex of $\Gamma$ together with a choice of the outer face is a planar embedding of $G$. A plane graph is a graph with a fixed planar embedding. A planar (plane) graph is maximal if each face of the graph is a triangle, thus no edge can be added to it without violating planarity. A graph $G$ is $k$-connected if it does not contain a set of $k-1$ vertices whose removal disconnects it.

Let $G=(V, E)$ be a maximal plane graph with outer face $\left(v_{1}, v_{2}, v_{n}\right)$. A canonical ordering [5] of $G$ is an order $\sigma=\left(v_{1}, v_{2}, v_{3}, \ldots, v_{n}\right)$ of its vertices satisfying the following properties: (1) The subgraph $G_{i-1}$ induced by $v_{1}, v_{2}, \ldots, v_{i-1}$ is 2 -connected and the boundary of the outer face of $G_{i-1}$ is a cycle $C_{i-1}$ containing edge $\left(v_{1}, v_{2}\right)$; (2) vertex $v_{i}$ is in the outer face of $G_{i}$ and its neighbors in $G_{i-1}$ form a (non-trivial) subpath of path $C_{i-1}-\left(v_{1}, v_{2}\right)$.

Let $G$ be a planar graph with a planar drawing $\Gamma$. Let $t_{1}$ and $t_{2}$ be two disjoint triangles of $G$. We say that $t_{2}$ is nested in $t_{1}$, and write $t_{2}<t_{1}$, if $t_{2}$ is in the bounded region of the plane delimited by $t_{1}$. A nested-triangles graph $G$ with $n$ vertices ( $n$ is a multiple of 3 ) is a 3 -connected graph admitting a planar drawing $\Gamma$ in which $n / 3$ disjoint triangles $t_{1}, t_{2}, \ldots, t_{n / 3}$ exist such that $t_{1}>t_{2}>\cdots>t_{n / 3}$.

\section{A Universal Point Subset for Planar Graphs}

In this section we provide a universal point subset of size $\lceil\sqrt{n}\rceil$ for (maximal) planar graphs with $n$ vertices. Note that considering maximal planar graphs is not a limitation, since any planar graph is a subgraph of a maximal planar graph.

Let $G$ be a maximal planar graph with $n$ vertices. Let $\sigma=\left(v_{1}, v_{2}, \ldots, v_{n}\right)$ be a canonical ordering of the vertices of $G$ for some planar embedding of $G$. Let $G_{i}$ be the subgraph of $G$ induced by the first $i$ vertices in $\sigma$ and let $C_{i}$ be the outer face of $G_{i}$. Bose et al. [2] define the frame $G^{\sigma}$ of $G$ with respect to $\sigma$ to be a directed subgraph of $G$ with edges: $\left(v_{1}, v_{2}\right)$ and, for every $v_{i}(i \geq 3),\left(v_{a(i)}, v_{i}\right)$ and $\left(v_{i}, v_{b(i)}\right)$ where $v_{a(i)}$ is the first and $v_{b(i)}$ the last vertex that are adjacent to $v_{i}$ on path $C_{i-1}-\left(v_{1}, v_{2}\right)$.

Let $<_{\sigma}$ be the partial order on the vertices of $G$ where $u<_{\sigma} v$ if and only if $G^{\sigma}$ contains a path from $u$ to $v$. Notice that $v_{a(i)}$ is the smallest vertex and $v_{b(i)}$ is the largest vertex according to $<_{\sigma}$ that are adjacent to $v_{i}$ in $G$ and precede $v_{i}$ in $\sigma$. A sequence of numbers $\left(x_{1}, x_{2}, \ldots, x_{n}\right)$ obeys the partial order $<_{\sigma}$ if $x_{a}<x_{b}$ for all $v_{a}<{ }_{\sigma} v_{b}$.

Lemma 1. Given a canonical ordering $\sigma=\left(v_{1}, v_{2}, \ldots, v_{n}\right)$ of the vertices of a maximal planar graph $G$ and a sequence of $x$-coordinates $\left(x_{1}, x_{2}, \ldots, x_{n}\right)$ that obeys the partial order $<_{\sigma}$ with $x_{i} \in[1, n]$, for any sequence of $y$-coordinates $\left(y_{1}, y_{2}, \ldots, y_{n}\right)$ satisfying $y_{1}=y_{2}=0$ and $y_{i}>\frac{n-1}{\Delta} y_{i-1}$ for $i \geq 3$, where $0<\Delta \leq \min _{v_{a}<_{\sigma} v_{b}} x_{b}-$ $x_{a}$, the drawing of $G$ with $v_{i}$ at point $\left(x_{i}, y_{i}\right)$ for all $i \in[n]$ is a plane drawing.

Proof: Suppose that the drawing of $G_{i-1}$ with $v_{j}$ at point $\left(x_{j}, y_{j}\right)$ for $j \in[i-1]$ is a plane drawing, and furthermore that $C_{i-1}$ is an $x$-monotone chain. Clearly, this holds for $i-1=2$. If the vertex $v_{i}$ at point $\left(x_{i}, y_{i}\right)$ lies in the intersection of the half-planes above the lines defined by consecutive vertices on the chain, then $v_{i}$ can connect to any 
subsequence of chain vertices without intersecting the drawing of $G_{i-1}$. By adding $v_{i}$ at $\left(x_{i}, y_{i}\right)$, we obtain a plane drawing of $G_{i}$ since $\left(v_{1}, v_{2}, \ldots, v_{n}\right)$ is a canonical ordering. Since the sequence $\left(x_{1}, x_{2}, \ldots, x_{n}\right)$ obeys the partial order $<_{\sigma}, C_{i}$ is $x$-monotone.

It remains to show that $\left(x_{i}, y_{i}\right)$ is above the lines through every pair of adjacent vertices in $C_{i-1}$. Let $v_{a}$ precede $v_{b}$ on the chain $C_{i-1}$. Since $v_{a}<_{\sigma} v_{b}, x_{a}<x_{b}$. The point $\left(x_{i}, y_{i}\right)$ lies above the line through $\left(x_{a}, y_{a}\right)$ and $\left(x_{b}, y_{b}\right)$ if $y_{i}\left(x_{b}-x_{a}\right)>$ $y_{a}\left(x_{b}-x_{i}\right)+y_{b}\left(x_{i}-x_{a}\right)$. By choosing $y_{i}>\frac{n-1}{\Delta} y_{i-1}$ this inequality holds for any $x_{i} \in[1, n]$, since $x_{b}-x_{a} \geq \Delta, y_{a}, y_{b} \leq y_{i-1}$, and $x_{a}, x_{b} \in[1, n]$.

Let $\mathcal{U}_{k}=\left\{\left((2 n)^{-n i},(2 n)^{n i}\right) \mid i \in[k]\right\}$ be a nearly vertical set of $k$ points in convex position. Observe that $\mathcal{U}_{k}$ is a one-sided convex set.

Lemma 2. If a maximal planar graph $G$ has a canonical ordering $\sigma$ so that $<_{\sigma}$ has an anti-chain of size $k$, then $G$ admits a planar straight-line drawing with $k$ of its vertices placed on $\mathcal{U}_{k}$.

Proof: Let $v_{1}, v_{2}, \ldots, v_{n}$ be the vertices of $G$ in canonical order $\sigma$. Let $A=\left\{v_{i_{1}}\right.$, $\left.v_{i_{2}}, \ldots, v_{i_{k}}\right\}$ be an anti-chain in $<_{\sigma}$ with $i_{1}<i_{2}<\cdots<i_{k}$. Note that $i_{1}>2$ (unless $k=1$ ) since $v_{1}$ and $v_{2}$ cannot be part of an anti-chain of size greater than one: $v_{1}<_{\sigma} v$ for all $v \neq v_{1}$ and $v<_{\sigma} v_{2}$ for all $v \neq v_{2}$. Let $A^{*}$ be the set of vertices less than (according to $<_{\sigma}$ ) some vertex in $A$. We create a sequence of $x$-coordinates $\left(x_{1}, x_{2}, \ldots, x_{n}\right)$ that obeys the partial order $<_{\sigma}$ with each $x_{i}$ an integer in $[1, n]$ for $v_{i} \notin A$ and $x_{i_{j}}=|S|+1+(2 n)^{-n j}$ for all $j \in[k]$. This is easy to achieve using a topological sort of $A^{*}$ and a topological sort of $V \backslash\left(A^{*} \cup A\right)$.

We create a sequence of $y$-coordinates $\left(y_{1}, y_{2}, \ldots, y_{n}\right)$ with $y_{1}=y_{2}=0$ and $y_{i}=(2 n)^{j n+\left(i-i_{j}\right)}$ for $i_{j} \leq i<i_{j+1}$ where, for convenience, we have assumed $i_{0}=1$ and $i_{k+1}=n+1$. This assigns the $j$ th vertex in the anti-chain a $y$-coordinate of the form $(2 n)^{j n}$, and it assigns vertices not in the anti-chain, that are between the $j$ th and $(j+1)$ th anti-chain vertices (in the canonical ordering $\sigma), y$-coordinates between $(2 n)^{j n}$ and $(2 n)^{(j+1) n}$. Since no two vertices in $A$ are related by $<_{\sigma}$, the minimum of $x_{b}-x_{a}$ for $v_{a}<_{\sigma} v_{b}$ is at least $1-(2 n)^{-n}>1 / 2=\Delta$. Thus the sequence $\left(y_{1}, y_{2}, \ldots, y_{n}\right)$ satisfies the conditions of Lemma 1. By that lemma, there is a plane drawing of $G$ with these $x$ - and $y$-coordinates. Shifting this drawing by $-\left|A^{*}\right|-1$ in the $x$-coordinate places the anti-chain $A$ on the points $\mathcal{U}_{k}$.

Lemma 3. Let $\pi$ be a maximal chain of $<_{\sigma}$. Then, the subgraph of $G$ induced by the vertices of $\pi$ is outerplanar.

Proof: Since $\pi$ is a maximal chain, it corresponds to a directed path, $P$, in $G^{\sigma}$ from $v_{1}$ to $v_{2}$. Let $C$ be the undirected cycle in $G$ composed of $P$ and the edge $\left(v_{1}, v_{2}\right)$. We prove that all the chords of $C$ in $G$ lie inside it with respect to the embedding used to derive the canonical ordering $\sigma$. Assume, for a contradiction, that $C$ has a chord $(u, v)$ outside $C$, where $u$ occurs before $v$ on $P$. Let $P^{\prime}=(u, w, \ldots, z, v)$ be the subpath of $P$ from $u$ to $v$. Suppose that $u$ precedes $v$ in $\sigma$. Since $(u, v)$ is an outside chord, the vertices in $P^{\prime}-v$ precede $v$ in $\sigma$. However, the fact that both $u$ and $z$ precede $v$ in $\sigma$ and $u<_{\sigma} z$ contradicts the fact that $(z, v)$ is an edge of $G^{\sigma}$. Indeed, $G^{\sigma}$ contains only one directed edge to $v$ from a vertex that precedes $v$ in $\sigma$. The edge is from the first vertex in $<_{\sigma}$ among the neighbors of $v$ in $G$ that precede $v$ in $\sigma$. Since $u<_{\sigma} z$ and since $u$ 


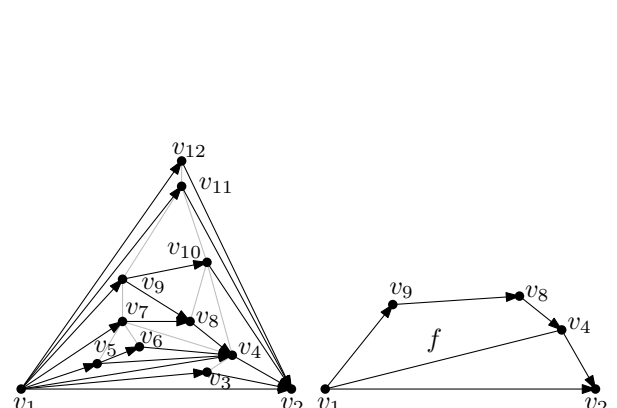

(a)

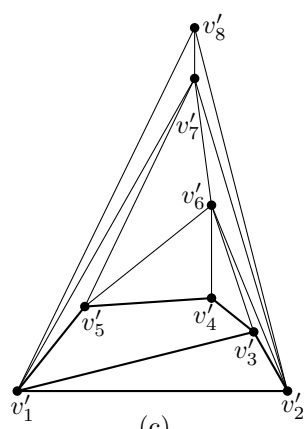

(c)

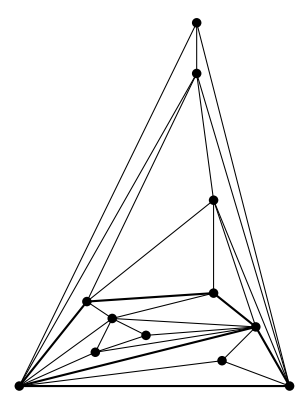

(d)

Fig. 1. (a) A maximal planar graph $G$. The edges of $G^{\sigma}$ are directed and black, while edges not in $G^{\sigma}$ are gray. $\pi=\left(v_{1}, v_{9}, v_{8}, v_{4}, v_{2}\right)$ is a maximal chain of $<_{\sigma}$. (b) A drawing of the subgraph $G(\pi)$ of $G$ on a one-sided convex point set. (c) Extending the drawing of $G(\pi)$. (d) The final drawing after filling in the faces of $G(\pi)$.

precedes $v$ in $\sigma$, vertex $z$ cannot be the first neighbor. Hence, edge $(z, v)$ cannot be in $G^{\sigma}$. Similarly, if $v$ precedes $u$ in $\sigma$, both $v$ and $w$ precede $u$, and $w<_{\sigma} v$ contradicts the fact that $(u, w)$ is an edge of $G^{\sigma}$.

Lemma 4. If a maximal planar graph $G$ has a canonical ordering $\sigma$ such that $<_{\sigma}$ has a maximal chain $\pi$ of size $k$, then $G$ admits a planar straight-line drawing with $k$ of its vertices placed on any one-sided convex set of size $k$.

Proof: Consider any one-sided convex point set $S$ of size $k$. Assume, without loss of generality up to a rotation of the coordinate system, that such points are ordered based on their $x$-coordinate and that the leftmost and the rightmost points are also the bottommost ones. By Lemma 3, the subgraph $G(\pi)$ of $G$ induced by the vertices of $\pi$ is outerplanar. Hence, such a subgraph can be drawn [1] on the points of $S$ in such a way that the vertices of $\pi$ are assigned increasing $x$-coordinates according to the order they appear on $\pi$. Figure 1(a) illustrates a maximal planar graph $G$, together with the frame $G^{\sigma}$ associated with a partial order $<_{\sigma}$ of $G$. Figure $1(\mathrm{~b})$ illustrates a drawing of the subgraph $G(\pi)$ of $G$ on a one-sided convex point set.

Further, consider the planar graph $G^{\prime}$ obtained from $G$ by removing every vertex that is internal to some face of $G(\pi)$. Since $G(\pi)$ is outerplanar, there exists a canonical ordering $\sigma^{\prime}$ of the vertices of $G^{\prime}$ such that the $k$ vertices of $G(\pi)$ appear in the first $k$ positions of $\sigma^{\prime}$, that is, $G(\pi)=G_{k}^{\prime}$. Since the drawing of $G(\pi)=G_{k}^{\prime}$ obtained by placing its vertices on $S$ is such that $C_{k}^{\prime}$ is an $x$-monotone chain, such a drawing can be extended to a planar drawing of $G^{\prime}$ by applying an algorithm that is analogous to the one given by de Fraysseix, et al. [5] to construct polynomial area drawings of planar graphs. Namely, place each vertex $v_{j}^{\prime}$ of $G^{\prime}$, with $j>k$, in such a way that $G_{j}^{\prime}$ is an $x$-monotone chain. Note that this is always possible, since vertices $v_{j}^{\prime}$, with $j>k$, do not need to be placed on prescribed points (neither a point of the prescribed point set nor an integer grid point, as it happens in [5]). See Fig. 1(c). 
Finally, for each face $f$ of $G(\pi)$, consider the subgraph $G_{f}$ of $G$ induced by the vertices of $f$ and by the vertices that are internal to $f$. Then, apply Tutte's algorithm [19, $20]$ to construct a planar drawing $\Gamma_{f}$ of $G_{f}$ such that the outer face of $G_{f}$ is represented in $\Gamma_{f}$ by the polygon delimiting $f$ in the drawing of $G(\pi)$ obtained by placing its vertices on $S$. Again, such a drawing can always be constructed since the internal vertices of $G_{f}$ do not need to be placed on prescribed points. The final drawing of the graph in Fig. 1(a) is depicted in Fig. 1(d).

The following theorem follows from Lemmas 2 and 4.

Theorem 1. There exists a set of $\lceil\sqrt{n}\rceil$ points that is a universal point subset for planar graphs with $n$ vertices.

Proof: Let $H$ be any planar graph. By adding edges to $H$, we obtain a maximal planar graph $G$. Let $\sigma$ be a canonical ordering of $G$ for some planar embedding of $G$. Let $G^{\sigma}$ be a frame of $G$ and let $<_{\sigma}$ be the corresponding partial order. By Dilworth's theorem [6], there exists in $<_{\sigma}$ either a chain of $\lceil\sqrt{n}\rceil$ vertices or an anti-chain of $\lceil\sqrt{n}\rceil$ vertices. In either case, by Lemma 2 or $4, G$ admits an embedding-preserving planar straightline drawing with $k=\lceil\sqrt{n}\rceil$ of its vertices placed on the points of the one-sided convex point set $\mathcal{U}_{k}=\left\{\left((2 n)^{-n i},(2 n)^{n i}\right) \mid i \in[k]\right\}$. Removing the added edges gives a drawing of $H$.

\section{Universalizing the Size of Universal Point Subsets}

Let $\mathcal{G}$ be a class of planar graphs. We define $F(n, \mathcal{G})$ as the maximum value such that every set of $F(n, \mathcal{G})$ points in general position is a universal point subset for the graphs in $\mathcal{G}$ with $n$ vertices. When $\mathcal{G}$ coincides with the class of all planar graphs, we simply denote this value by $F(n)$. In this section we give lower and upper bounds for $F(n, \mathcal{G})$ for some classes of planar graphs.

\subsection{Planar graphs}

When $\mathcal{G}$ coincides with the class of all planar graphs, we show that $F(5)=3, F(6)=4$, and $4 \leq F(n) \leq 2\left\lceil\frac{n}{3}\right\rceil+1$ for all the other values of $n$.

First observe that there exists a set of 4 points in general position that is not a universal point subset for planar graphs with $n=5$ vertices. Indeed, consider the set $S$ of 4 points at the corners of a unit square. The claim follows from the fact that the outer face of every maximal planar graph with 5 vertices can use at most one point of $S$, as otherwise it could not contain all the remaining points of $S$ in its interior. This, together with the fact that, by Tutte's theorem $[19,20]$, every set of 3 points in general position is a universal point subset for planar graphs, implies that $F(5)=3$. Then, we consider planar graphs with $n=6$. Again, with the same argument as the one used for $n=5$, we can prove that a set of 5 points composed of the corners of a regular pentagon is not a universal point subset for all planar graphs of size 6 , which means $F(6) \leq 4$. In the following lemma we prove that also this bound is tight (that is, $F(6)=4$ ). First, we note that all maximal planar graphs with six vertices are those depicted in Fig. 2(a-d). 


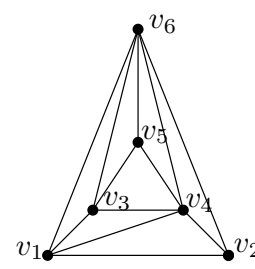

(a)

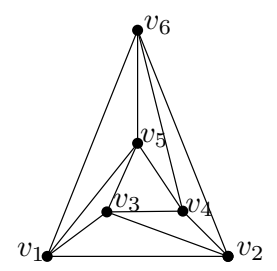

(b)

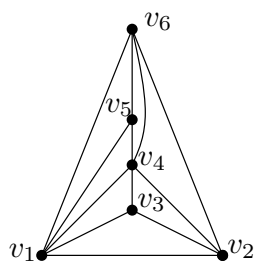

(c)

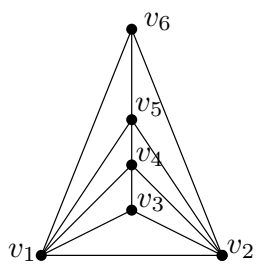

(d)

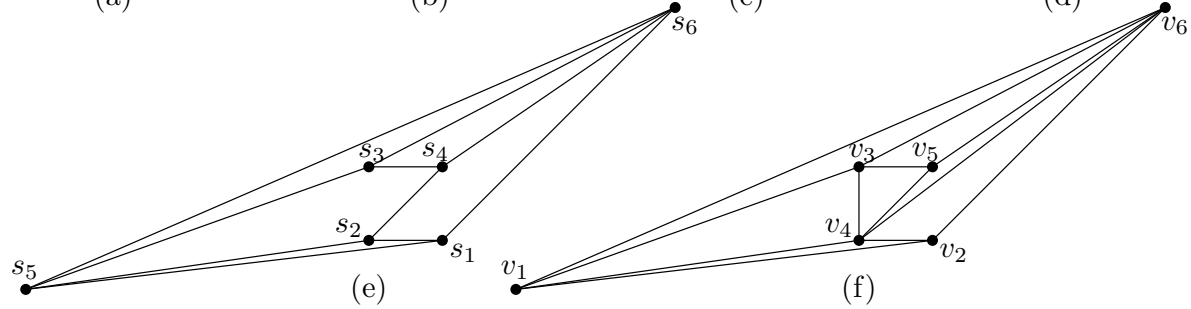

(e)

(f)

Fig. 2. (a-d) All maximal six-vertex planar graphs. (e) The common structure used to draw them on a set of four points in convex position. (f) The corresponding drawing of the graph in (a).

Lemma 5. $F(6)=4$.

Proof: Let $S$ be a set of 4 points in general position. If $S$ is not in convex position, we map the vertices of the outer face of $G$ to the three points on the convex hull $C H(S)$ of $S$. For all cases of Fig. 2(a-d), the remaining vertices are drawn inside $C H(S)$, by using the fourth point of $S$ to place one of them.

If the points of $S$ are in convex position, let $s_{1}, s_{2}, s_{3}$, and $s_{4}$ be the points of $C H(S)$ in clockwise order. Let $s_{5}$ and $s_{6}$ be points not in $S$ so that triangle $s_{1} s_{5} s_{6}$ contains $S \backslash\left\{s_{1}\right\}$ in its interior; $s_{5}$ sees $s_{3}, s_{4}, s_{2}$, and $s_{1}$ in clockwise order; and $s_{6}$ sees $s_{3}, s_{4}, s_{2}$, and $s_{1}$ in counterclockwise order. It is straightforward to confirm that such points exist since $S$ is in convex position. Add segments $s_{1} s_{2}, s_{2} s_{4}, s_{4} s_{3}, s_{5} s_{6}$, $s_{5} s_{3}, s_{5} s_{2}, s_{5} s_{1}, s_{6} s_{3}, s_{6} s_{4}$, and $s_{6} s_{1}$. See Fig. 2(e). For each case of Fig. 2(a-d), we map the outer vertices of $G$ to the points $s_{1}, s_{5}, s_{6}$, we map the internal vertices of $G$ to the points $s_{2}, s_{3}, s_{4}$ and we insert the remaining edges of $G$. As an example, the drawing of the graph in Fig. 2(a) is shown in Fig. 2(f).

Finally, we consider the general case, namely planar graphs with $n>6$ vertices. We first observe that, by using an argument similar to the one used to prove $F(5) \leq 3$ and $F(6) \leq 4$, we can prove that $F(n) \leq n-2$. Indeed, a set of $n-1$ points composed of the corners of a regular $(n-1)$-gon is not a universal point subset for all maximal planar graphs of size $n$, as at most one point in this point set can be used to place vertices of the outer face. However, as shown in the following theorem, in the general case we can prove a better upper bound. On the other hand, the lower bound of 4 is obtained by extending the result for planar graphs with six vertices.

Theorem 2. If $n>6$ then $4 \leq F(n) \leq 2\left\lceil\frac{n}{3}\right\rceil+1$.

Proof: We first prove the lower bound. Let $S$ be a set of 4 points in general position. Consider a maximal planar graph $G$ with $n \geq 6$ vertices. The proof is by induction on 
the number of vertices of $G$. In the base case $G$ has $n=6$ vertices, and the statement follows from Lemma 5. For $n>6$, we can use Read's algorithm [14] to produce a straight-line drawing of $G$ given a planar embedding of $G$. Let $u$ be an internal vertex of $G$ and denote by $N(u)$ the set of neighbors of $u$. We can have three cases: (i) if $\operatorname{deg}(u)=3$, then remove $u$; (ii) if $\operatorname{deg}(u)=4$, then let $v \in N(u)$ be a vertex with exactly two neighbors in $N(u)$ : remove $u$ and triangulate the quadrilateral face by adding an edge $(v, x)$ such that $x \in N(u)$ and $x \notin N(v)$; (iii) if $\operatorname{deg}(u)=5$, then let $v \in N(u)$ be a vertex with exactly two neighbors in $N(u)$ : remove $u$ and triangulate the pentagonal face by adding two edges $(v, x)$ and $(v, y)$ such that $x, y \in N(u)$ and $x, y \notin N(v)$. Denote by $G^{\prime}$ the reduced graph. Note that, by Euler's formula, $G$ always contains a vertex of degree 3,4 , or 5 . Moreover, $G^{\prime}$ and $G$ have the same outer face and in all the above cases the neighbors of $v$ in $G^{\prime}$ that are neighbors of $u$ in $G$ occur consecutively in clockwise order around $v$. Since $G^{\prime}$ has $n-1$ vertices and it is maximal planar, $S$ is a universal point subset for $G^{\prime}$ by induction. Let $\Gamma^{\prime}$ be an embedding preserving drawing of $G^{\prime}$ that uses the points of $S$. Consider the cycle $C$ composed by the vertices that were adjacent to $u$ before its removal. Depending on $\operatorname{deg}(u), C$ can be a triangle, a quadrilateral, or a pentagon. For each of these cases, by construction, there exists a point $p$ inside $C$ sufficiently close to $v$ on which we can draw $u$ and obtain an embedding preserving drawing of $G$ [14].

Now we prove the upper bound. Let $G$ be a graph with $n>6$ vertices that contains $\lfloor n / 3\rfloor$ nested triangles. Let $S$ be a set of $n-\left(\left\lfloor\frac{n}{3}\right\rfloor-2\right) \leq 2\left\lceil\frac{n}{3}\right\rceil+2$ points in convex position. All but $\lfloor n / 3\rfloor-2$ vertices of $G$ must map to points of $S$. Thus, there are at least two nested triangles $t_{1}$ and $t_{2}$ of $G$ that have all three vertices mapped on points of $S$. However, since $S$ is in convex position, $t_{1}$ cannot include $t_{2}$ and vice-versa.

\subsection{4-connected planar graphs}

Next, we consider the value of $F(n, \mathcal{G})$ when $\mathcal{G}$ coincides with the class of 4-connected planar graphs whose outer face is a 4 -cycle. For this class we can prove a stronger lower bound than for planar graphs, namely $F(n, \mathcal{G}) \geq \frac{\lg n}{4}$.

Theorem 3. Let $\mathcal{G}$ be the class of 4-connected planar graphs whose outer face has size at least 4 . Then, $\frac{\lg n}{4} \leq F(n, \mathcal{G}) \leq n-2$.

Proof: The upper bound comes analogously to $F(n) \leq n-2$ for planar graphs. In fact, consider a 4-connected planar graph with $n>8$ vertices and whose outer face is a 4 -cycle, and consider a point set $S$ composed of the corners of a regular $(n-1)$-gon. Again, at least two points not in $S$ have to be used to place the vertices of the outer face.

We prove the lower bound. Let $S$ be any set of $\left\lceil\frac{\lg n}{4}\right\rceil$ points in general position. Let $G$ be an internally 4-connected plane graph with $n$ vertices and outer face of size at least 4. Thomassen [17] showed that $G$ is the dual of a rectangular subdivision of a rectangle. Tóth [18] showed that there exists a horizontal or vertical line (called a stabber) that intersects at least $\frac{\lg n}{4}$ rectangles in this rectangular subdivision. Find such a stabber that intersects rectangles $r_{1}, r_{2}, \ldots, r_{k}\left(k \geq \frac{\lg n}{4}\right)$. Add points to $S$ to create a set $S^{\prime}$ of $k$ points. Choose axes so that no two points of $S^{\prime}$ have the same $x$-coordinate. Let $p_{1}, p_{2}, \ldots, p_{k}$ be the points of $S^{\prime}$ sorted by $x$-coordinate. Place the vertex corresponding 
to rectangle $r_{i}$ at $p_{i}$ for $i \in[k]$. So $S^{\prime}$ supports the $x$-monotone drawing of a path in $G$, and the vertices corresponding to $p_{1}$ and $p_{k}$ lie on the outer face of $G$. This path divides $G$ into two subgraphs $G_{1}$ and $G_{2}$, one on each side of the path. So we can use the construction by de Fraysseix, et al. [5] twice, once for $G_{1}$ and once for $G_{2}$.

\subsection{Nested-triangles graphs}

Finally, we consider the value of $F(n, \mathcal{G})$ when $\mathcal{G}$ coincides with the class of nestedtriangles graphs. We prove that for this class $F(n, \mathcal{G}) \geq \frac{n}{3}$, almost matching the upper bound.

Theorem 4. Let $\mathcal{G}$ be the class of nested-triangles graphs. Then, $\frac{n}{3} \leq F(n, \mathcal{G}) \leq$ $2\left\lceil\frac{n}{3}\right\rceil+1$.

Proof: The upper bound is the same proved in Theorem 2. As for the lower bound, let $S$ be any set of $\frac{n}{3}$ points in general position and choose the coordinate axes in such a way that no two points have the same $y$-coordinate. Let $G$ be a nested-triangles graph with a given planar embedding. Let $v$ be a vertex of the triangle $t$ representing the outer face of $G$ and let $p$ be the point of $S$ having the largest $y$-coordinate. Map $v$ to $p$ and represent $t$ as a triangle that encloses all remaining points of $S$. Remove the outer face of $G$ and repeat the argument on $S \backslash\{p\}$; at every step, the sides of the triangles that represent the outer face are drawn parallel to one another in such a way that the inclusion of the triangular faces is respected and no two edges cross.

\section{Final Remarks and Open Problems}

We remark that in this paper we assumed the points to be in general position. This is coherent with most of the literature in combinatorial and computational geometry, where geometric graphs (i.e. planar straight line drawings) are defined on point sets in general position [12]. However, one might also consider point sets allowing collinearities. In this scenario, some of our results should definitely be reformulated. For example, it is easy to see that a point set of four collinear points cannot be a universal point subset for the class of maximal planar graphs with six vertices. For a class $\mathcal{G}$ of planar graphs one could define $\bar{F}(n, \mathcal{G})$ as the maximum value such that every set of $\bar{F}(n, \mathcal{G})$ distinct points, whether it contains collinearities or not, is a universal point subset for the graphs in $\mathcal{G}$ with $n$ vertices, and analogously define $\bar{F}(n)$ for planar graphs. Note that, allowing collinearities makes it possible to relate the problem of determining the value of $\bar{F}(n)$ with the allocation problem for planar graphs [11,13]. In this problem, the input is an $n$-vertex planar graph $G$ and a point set $X$ of size $n$, possibly with collinearities, and the goal is to construct a planar drawing $\Gamma$ of $G$ such that as many vertices of $G$ as possible are placed in $\Gamma$ on points of $X$. In particular, by exploiting this relationship, a slightly sublinear upper bound can be proved for $\bar{F}(n)$ via a construction from [13] that makes heavy use of collinearity.

We conclude with a few open problems that we find particularly interesting. (i) Narrow the gaps between the upper and lower bounds of Section 3. (ii) Prove/disprove a sublinear upper bound for $F(n)$ when points are in general position. (iii) Does the $\lim _{n \rightarrow \infty} F(n)=\infty$ hold? (iv) Is there any universal subset for the set of all planar graphs with $n$ vertices that consists of more than $\lceil\sqrt{n}\rceil$ points? 


\section{References}

1. P. Bose. On embedding an outer-planar graph in a point set. Comp. Geom., 23(3):303 - 312, 2002.

2. P. Bose, V. Dujmovic, F. Hurtado, S. Langerman, P. Morin, and D. R. Wood. A polynomial bound for untangling geometric planar graphs. Discrete \& Comp. Geom., 42(4):570-585, 2009.

3. F.-J. Brandenburg. Drawing planar graphs on $\frac{8}{9} n^{2}$ area. Electronic Notes in Discrete Mathematics, 31:37-40, 2008.

4. S. Cabello. Planar embeddability of the vertices of a graph using a fixed point set is NP-hard. J. Graph Algor. and Applic., 10(2):353-363, 2006.

5. H. de Fraysseix, J. Pach, and R. Pollack. How to draw a planar graph on a grid. Combinatorica, 10(1):41-51, 1990.

6. R. Dilworth. A decomposition theorem for partially ordered sets. Annals of Mathematics, 51(1):161-166, 1950.

7. I. Fáry. On straight-line representation of planar graphs. Acta Sci. Math. (Szeged), 11:229$233,1948$.

8. J. E. Goodman and R. Pollack. Allowable sequences and order types in discrete and computational geometry. New Trends in Discrete and Comp. Geom., pages 103-134, 1993.

9. P. Gritzmann, B. Mohar, J. Pach, and R. Pollack. Embedding a planar triangulation with vertices at specified points. Amer. Math. Monthly, 98(2):165-166, 1991.

10. M. Kurowski. A 1.235 lower bound on the number of points needed to draw all $n$-vertex planar graphs. Inf. Process. Lett., 92(2):95-98, 2004.

11. A. G. Olaverri, F. Hurtado, C. Huemer, J. Tejel, and P. Valtr. On triconnected and cubic plane graphs on given point sets. Comput. Geom., 42(9):913-922, 2009.

12. J. Pach and P. K. Agarwal. Geometric graphs. In Comb. Geom., pages 223-239. Wiley, 1995.

13. A. Ravsky and O. Verbitsky. On collinear sets in straight-line drawings. In P. Kolman and J. Kratochvíl, editors, WG, volume 6986 of $L N C S$, pages 295-306, 2011.

14. R. Read. A new method for drawing a planar graph given the cyclic order of the edges at each vertex. Congressus Numeration, 56:31-44, 1987.

15. W. Schnyder. Embedding planar graphs on the grid. In D. S. Johnson, editor, SODA, pages 138-148. SIAM, 1990.

16. S. K. Stein. Convex maps. Proc. of the Amer. Math. Society, 2(3):464-466, 1951.

17. C. Thomassen. Interval representations of planar graphs. J. of Comb. Theory, Series B, 40(1):9-20, 1986.

18. C. D. Tóth. Axis-aligned subdivisions with low stabbing numbers. SIAM J. Discrete Math., 22(3):1187-1204, 2008.

19. W. T. Tutte. Convex representations of graphs. Proc. London Math Soc., 10:304-320, 1960.

20. W. T. Tutte. How to draw a graph. Proc. London Math Soc., 13:743-768, 1963.

21. K. Wagner. Bemerkungen zum vierfarbenproblem. Jahresbericht. German. Math.-Verein., 46:26-32, 1936.

Acknowledgments: Work on this problem began at the BICI Workshop on Graph Drawing, held in Bertinoro, Italy, in March 2012. We thank all the participants for many fruitful discussions. Research supported in part by the MIUR project "AlgoDEEP" prot. 2008TFBWL4 and by the ESF project 10-EuroGIGA-OP-003 GraDR "Graph Drawings and Representations". William Evans is partially supported by NSERC of Canada. Ferran Hurtado is partially supported by projects MICINN MTM2009-07242, Gen. Cat. DGR2009SGR1040, and ESF EUROCORES programme EuroGIGA, CRP ComPoSe: MICINN Project EUI-EURC-2011-4306. Yoshio Okamoto is partially supported by Grand-in-Aid for Scientific Research from Ministry of Education, Science and Culture, Japan and Japan Society for the Promotion of Science. 